deal of additional work remains to be done on the solution of equations and systems of equations.

David M. Young, JR.

Topology. By John G. Hocking and Gail S. Young. Addison-Wesley, Reading, Mass., 1961. $9+374$ pp. $\$ 8.75$.

This book is designed as a text for a one year first course in topology. Three chapters on general topology and a chapter on homotopy theory constitute the proposed first semester's course, four chapters on algebraic topology the second term's program. The authors have adopted a policy of including by mention or brief description many topics not covered extensively, with the object of providing orientation for further study.

The first two chapters proceed in more-or-less standard fashion with the study of the notion of a topology and the elementary properties of topological spaces: compactness, connectedness, separation and so forth. There is concordantly a discussion of metric spaces. The scope may be suggested by the principal theorems, viz. the Tychonoff compactness theorem, the Tietze extension theorem, the Urysohn metrization theorem and the Baire category theorem. Orientational material, treated perfunctorily and in general without proofs, includes function spaces, uniform structures, topological groups, paracompactness, the Smirnov metrization theorem, inverse limits.

The third chapter contains a more intensive study of compacta than is fashionable nowadays, with a proof of the Hahn-Mazurkiewicz theorem and discussion of monotone-light factorization and indecomposability. There is also a nod towards dimension theory.

Chapter four compresses a remarkably large amount of homotopy theory into 43 pages: homotopy of maps, Borsuk's homotopy extension theorem, essentiality, absolute homotopy groups, knot theory, covering spaces, homotopy local connectedness. Some of these subjects are of course only briefly mentioned. The knot theory is purely descriptive; covering spaces are honored by a definition and a few theorems stated without proof. But it may be fair to say that homotopy theory suffers from the compression. The treatment seems too dense to be readable at this level, and the choice of theorems proved is ill-calculated to give a coherent picture of the structure of the theory.

The next two chapters are devoted to the machinery of homology theory of simplicial complexes, i.e. to the geometry of polytopes and the construction of chain, cycle, boundary and homology groups. Chapter seven discusses relative homology and cohomology, and in- 
cludes a treatment of cup and cap products. Poincaré duality is stated without proof. The last chapter presents Čech homology, singular homology and some aspects of Alexander duality.

This seems, in a way, to be a reasonable introduction to algebraic topology; indeed many topologists are likely to regard it as perfectly satisfactory. Nevertheless I am compelled to record my own feeling of dissatisfaction with it.

Briefly, in spite of courteous gestures toward modern developments, the exposition of homology theory appears to be irreparably old-fashioned. Thus the Eilenberg-Steenrod axioms are listed butfor simplicial homology at least - the homotopy axiom is never proved. Thus invariance of cup and cap products remains unproved, and their connection with the diagonal map is left without notice. The topological invariance of the simplicial homology groups is proved by comparison with Cech theory. This is technically correct but surely misleading, since this invariance, and indeed homotopy invariance, is easily proved intrinsically.

The algebraic technique is similarly obsolete. Chain complexes are nowhere treated as independent entities; the connecting homomorphism and the exactness of the homology sequence must be developed separately in each of several examples. There is an old-fashioned proof of the universal coefficient theorem. The Künneth theorem is not even stated. Probably enough elementary homological algebra for these purposes could have been developed in the space devoted to ponderous computation with canonical bases.

Most of these difficulties stem from an essential insensitivity to the functorial character of the invariants of algebraic topology. A regard for this character is, I believe, so essential to the understanding of the field today that even the most elementary text must be so organized as to further the student's appreciation of it. This is not to say that a text in algebraic topology need be an exercise in categories and functors, but rather that the presentation must be governed by the author's consciousness of their role.

Against these strictures must be set the admirable tone of the book, which manages happily to reconcile the inclusion of a great deal of mathematics with the maintenance of a generally leisured manner, to keep always in sight the geometric motivation, and to illustrate the text liberally with examples and problems.

There are unfortunately a number of mistakes: as an example only, the definition of the Hausdorff metric on p. 42 is clearly wrong. Most of these are unimportant, though there are certainly too many of them. They should not cause serious difficulties, nor detract greatly 
from a high evaluation of the book, conditioned only by the considerations mentioned above.

\section{Alex Heller}

Introduction to complex analysis. By Zeev Nehari. Allyn and Bacon, Boston, Mass., 1961. 258 pp. $\$ 7.50$.

It is generally hard to balance a course in complex variables between the theory for the mathematics students and the applications for the engineers. Many universities solve this problem by giving two separate courses. Nehari offers another solution which draws a happy compromise between theory and applications. His book is designed for a one semester course in complex variables (the fashionable word is now complex "analysis") which prepares the interested student for further study in the theory of functions and which is also rich in applications. The book is written in Nehari's informal style which makes enjoyable reading. Embodied in the compromise mentioned above is naturally a sacrifice of generality in some of the basic definitions and theorems, but enough is always given to present a good idea of the general theory of analytic functions.

The chapter titles are: I. Complex variables, II. Analytic functions, III. Complex integration, IV. Applications of Cauchy's theorem, V. Conformal mapping, VI. Physical appliCations, ViI. Additional topics. All of the material that one would expect to find under each of the first six chapter headings is there. There are, however, many little embellishments which make this book especially good. From the very beginning, conjugate coordinates are introduced and used to simplify proofs. There is a nice presentation of the extensions of the elementary functions to the complex plane and their properties. An intuitive "scissors and paste" type of explanation of Riemann surfaces is given. Although the Riemann mapping theorem is stated without proof, the chapter on conformal mapping is especially rich in material for an introductory course. The section on the Schwarz-Christoffel formula is especially noteworthy. The physical applications include boundary value problems, electrostatic fields, and two-dimensional fluid flows. The topics in the last chapter are analytic continuation, the gamma and zeta functions, the symmetry principle, and methods for determining the harmonic conjugate using conjugate coordinates. The book is very richly endowed with stimulating exercises, some of which will certainly challenge the students.

George Springer 